\title{
Decreased Door to Balloon Time: Better Outcome for the Patient?
}

\author{
Steven B. Deutsch, Eric L. Krivitsky* \\ University of South Florida Morsani College of Medicine, Tampa, Florida, USA \\ Email: sbdeutsch@gmail.com
}

Received 19 January 2015; accepted 27 February 2015; published 5 March 2015

Copyright (C) 2015 by authors and Scientific Research Publishing Inc.

This work is licensed under the Creative Commons Attribution International License (CC BY). http://creativecommons.org/licenses/by/4.0/

(c) () Open Access

\begin{abstract}
Recently the American College of Cardiology and the American Heart Association instituted DTB of 90 minutes or less as a class I recommendation. Since 2006 the percentage of patient meeting this metric has substantially increased, although research has demonstrated discrepancies in whether or not this objective is associated with better patient outcome. Here, we reviewed seven studies in effort to investigate the validity of the $\mathbf{9 0}$ minute or less door to balloon time. Our findings suggest that patient outcome in the setting of acute myocardial infarction is multifactorial, and while the door to balloon time metric likely has played a role in better patient outcomes it is not the sole factor in improving mortality rates.
\end{abstract}

\section{Keywords}

Door to Balloon Time, 90 Minute Door to Balloon Time, Coronary Intervention, Myocardial Infarction

\section{Introduction}

683,000 patients in the United States are diagnosed with acute coronary syndrome (ACS) each year. ST elevation myocardial infarction (STEMI) comprises $25 \%$ - $40 \%$ of these individuals. The recommended approach of myocardial reperfusion is percutaneous coronary intervention (PCI) when it can be performed in a timely manner. Compared with other methods such as thrombolytic therapy, PCI reduces the risk of coronary reinfarction, increases the incidence of coronary patency in a previously infracted artery, as well as decreases the risk of hemorrhagic stroke associated with thrombolytics [1]. Over the last decade, there has been an emphasis on decreasing the door to balloon time (DTB) with the presumption that increased time correlates to increased mortality. This conclusion was deduced from compelling evidence brought forth by the Global Use of Strategies to Open Occluded Arteries in ACS as well as analysis from the National Registry of Acute MI. Both of these stu-

${ }^{*}$ Corresponding author. 
dies showed similar data in that the lowest mortality rate was observed in patients undergoing PCI earlier in their hospital course. Given the supposed time dependency of survival in patients with STEMI, the American College of Cardiology and the American Heart Association instituted DTB of 90 minutes or less as a class I recommendation in 2013. Per the new 2013 guidelines, it is emphasized that a decreased DTB is associated with a decrease in hospital mortality [2].

In 2005 the percentage of patients who had a DTB less than 90 minutes was roughly $45 \%$. Over the years, secondary to convincing evidence as well as quality improvement initiatives, the percentage of patients achieving a DTB time of less than 90 minutes is greater than $90 \%$. Despite this remarkable result, it is still questionable whether the focus and resulting change in DTB has led to a decrease in mortality in our post STEMI/PCI patient population based on existing research [3].

\section{Purpose}

The purpose of this study is to investigate whether a 90 minute DTB metric improves patient outcome.

\section{Hypothesis}

Given the AHA and ACC class I recommendation of a DTB of less than or equal to 90 minutes we hypothesize that review of current data should show that a more rapid time to coronary artery reperfusion will result in a decreased mortality in the STEMI/PCI patient population.

\section{Methods}

We reviewed seven studies consisting of cohort analyses, observational studies, prospective observational studies as well as prospective cohort analyses through the years 1984-2003, 1994-1998, 1999-2002, and 2005-2006, 2014 using search words such as "Door to balloon Time", "Association of Mortality and Door to Balloon Time”, "Percutaneous Intervention and Door to Balloon Time", "ST Elevation Myocardial Infarction and Door to Balloon Time” in PubMed, National Center for Biotechnology Information, Journal of American Medical Association, and the American Heart Association databases.

\section{Results}

Menees et al. analyzed data from the CathPCI Registry which took into account records from 515 hospitals. 96,738 admissions of STEMI patients undergoing PCI were analyzed and the DTB was evaluated. This study excluded patients who had been transferred from another facility for primary PCI and those who were undergoing nonemergency PCI. Also excluded, were patients for whom the door-to-balloon times were longer than 3 hours, in an effort to include the patients who had the most to gain with respect to myocardial salvage. They found that although DTB had decreased from 83 minutes from July 2005 through June 2006 to 67 minutes from July 2008 through June $2009(P<0.001)$, there was no statistically significant change in in-hospital mortality (4.8\% compared to $4.7 \%, P=0.43$ ). There was also a non-significant reduction in risk adjusted mortality from $5.0 \%$ to $4.7 \%(P=0.34)$. The authors discussed that the study patients had numerous clinical, procedural, and demographic differences between the patients enrolled with a therefore significant risk of confounding factors. The authors further suspected that additional reduction in DTB would be unlikely to improve hospital mortality. The results emphasized that a decreased DTB may be associated with decreased long term mortality, decreased hospital admission for heart failure and improved left ventricular function. Menees et al. further believed that DTB is one factor of total ischemic time, making the time before arrival to the hospital a more important constituent. Efforts with potential to improve outcomes may include increasing patient awareness of symptoms [4].

The beneficial effect of early reperfusion therapy in STEMI hinges on the concept of myocardial salvage of viable cardiac muscle. Brodie et al. specifically found that in patients with acutely decreased left ventricular ejection fraction, improvements after reperfusion were greatest in early reperfusion, $(6.9 \% \pm 11 \%$ at $<2$ hours vs. $3.1 \% \pm 12 \%$ when $>2$ hours; $P=0.007$ ) indicating that reperfusion performed within a 2-hour window is of paramount importance in ensuring left ventricular recovery. Outside of this window, ventricular recovery is modest at best and survival becomes largely independent of DTB time. This study population was taken from 2322 consecutive patients with STEMI treated with primary PCI without previous thrombolytic therapy at our institution from 1984 through 2003. Patients with chest pain of $<12 \mathrm{~h}$ duration or $>12 \mathrm{~h}$ for persistent pain or hemodynamic compromise and with electrocardiographic ST-segment elevation $\geq 1 \mathrm{~mm}$ in $\geq 2$ contiguous leads or left 
bundle branch block and without severe co-morbid disease were selected for intervention [5]. Schomig et al. upheld this relationship between decreased DTB time and improved myocardial salvage as measured by scintigraphy. They also concluded that PCI was superior to thrombolysis, independent of specific time to treatment intervals [6]. In another study, Brodie et al. evaluated two large trials, HORIZONS-AMI and CADILLAC trials and data clearly depicts the superior one year survival seen in patients with DTB times of less than 90 minutes, further substantiating the 2 hour window for myocardial salvage. Moreover, this mortality benefit persisted regardless of the perceived risk of the patient (risk defined by ACC/AHA as anterior/septal location of MI, DM, tachycardia $>100$, SBP $<100 \mathrm{mmHg}$ ) [7].

McNamera et al. used an acute MI registry in San Francisco to develop a cohort of 29,222 STEMI patients reperfused by PCI and noted that symptom onset to presentation was not significantly associated with in-hospital mortality. In this study, the following exclusion criteria were taken into account; patients that were transferred to or from another acute care institution $(\mathrm{n}=341,730)$ with neither ST-segment elevation in two plus echocardiogram leads, nor left bundle branch block on the first electrocardiogram $(n=334,013)$. Symptom onset after the admission date and time $(n=4305)$; with a non diagnostic first ECG, meaning the first ECG did not show ST-segment elevation or left bundle branch block; $n=14,314$ ); with diagnostic ECG that preceded hospital presentation by more than one hour (pre-hospital ECG), or with time from door-to-diagnostic ECG that was more than $6 \mathrm{~h}$ or missing ( $n=6467)$; who did not receive primary PCI $(n=92,772)$; with door-to-balloon times that were negative, more than six hours, or missing $(n=925)$; and with unknown time of symptom onset $(n=4804)$. In addition, to avoid including hospitals that performed primary PCI uncommonly, patients treated in hospitals reporting fewer than 20 PCI patients over the four-year time period $(n=1921)$ were excluded. The final cohort included 29,222 patients from 395 hospitals. Mortality status at the time of discharge was known for all patients. This study demonstrated longer DTB times, however, were associated with poorer outcomes (mortality rate of $3.0 \%, 4.2 \%$, 5.7\%, 7.4\% for DTB < 90 minutes, 91 - 120 minutes, 120 to 150 minutes and $>150$ minutes respectively; $P<$ 0.01) regardless of the duration of symptom onset to door time [8] [9]. It is important to note that the "time of symptom onset" factor was obtained from patient history, and may have been an inaccurate representation, hence influencing the results. Additionally, other patient risk factors may have not been considered, although influencing DTB. In this study, most patients were treated with DTB greater than that recommended in the guidelines. Data from the gold standard DTB of less than 90 minutes were not incorporated in the study results.

Cannon et al. tested their hypothesis that more rapid time to reperfusion in patients presenting with myocardial infarction results in lower mortality in the strategy of primary angioplasty. This group used an observational study of 767,409 patients that were enrolled in the NRMI-2 study. MI was defined as patient history suggestive of MI supplemented by creatinine kinase or CK-MB at least two times the upper limit of normal or electrocardiographic evidence of MI. If the prior listed criteria were inconclusive, scintigraphic, alternative enzymatic or echocardiographic evidence indicating MI qualified patients into the study. It was concluded that in a cohort of more than 27,000 patients treated with primary angioplasty, a DTB longer than 2 hours was an important factor related to mortality. There results showed a mortality rate of $4.9 \%$ with a DTB of $0-2 \mathrm{hrs}, 5.2 \%>2-3 \mathrm{hrs}$, $6.5 \%>3-4 \mathrm{hrs}, 6.7 \%>4-6 \mathrm{hrs}$, and $6.9 \%>6-12 \mathrm{hrs}$ with a $P$-value of $<0.001$. Cited weaknesses associated with this study relate to its study design: it was observational and the patients were not randomized to rapid versus slower DTB. The patients in this study were voluntary and there was no on-site monitoring of the data. The majority of hospitals in this prospective, observational study are relatively low volume primary angioplasty sites, and therefore, it is quite possible that conclusions from this study may not correlate to that of high volume skilled angioplasty centers [10].

The advantages of a decreased DTB in patients with STEMI were highlighted in Rathore et al. Their efforts demonstrated that any delay in PCI resulted in an increased mortality in hospital, even amongst patients treated within 90 minutes. Their prospective cohort study analyzed 43,801 subjects that were enrolled in the American College of Cardiology National Cardiovascular Data Registry in 2005-2006. Exclusion criteria limited study analysis to patients who, in 2005-2006, presented to a participating center within 12 hours of symptom onset with laboratory and electrocardiographic evidence of ST elevation myocardial infarction and subsequently underwent primary percutaneous coronary intervention $(n=64,676)$. Patients who were transferred from other hospitals $(n=17,992)$, as well as patients that first received fibrinolytic therapy and were subsequently referred for primary percutaneous coronary intervention $(n=3313)$. Patients under 18 years or over 99 years $(n=9)$ were excluded to focus analysis on adult patients suitable for primary percutaneous coronary intervention. Finally patients treated at hospitals that reported fewer than five primary percutaneous coronary interventions $(\mathrm{n}=29)$.

Rathore reported a median DTB of 83 minutes, with $57.9 \%$ of patient being treated within 90 minutes. The 
patients were divided into four separate groups for the purpose of evaluating the differences in patient characteristics associated with time to treatment. Their statistical analyses revealed similar trends to the McNamera et al study previously described. In DTB of 30 minutes, the in-hospital mortality was $3.0 \%$, 60 minutes $=3.5 \%$, 90 minutes $=4.3 \%, 120$ minutes $=5.6 \%, 150$ minutes $=7.0 \%, 180$ minutes $=8.4 \%$ with a $P<0.001$ [11]. Noted limitations of this study were the inability to assess the association of onset of patient symptoms to their arrival at a hospital and mortality or the association of total ischemic time and mortality. An additional limitation of this investigation was the failure to comment on the relationship of DTB and mortality at later end points.

\section{Conclusion}

Research investigating DTB has shown conflicting results on timing associated with mortality. From the studied investigations, we believe that DTB does indeed have a role in decreasing mortality, although it is not the only factor that comes into play. We do not believe that 90 minutes is the optimal number, as much as time is muscle. A delay in patient care, be it prior to presenting to the hospital door or while inside a health care center must be confronted. Door to balloon time comprises a part of the overall health care system delay [12]. Door to balloon time patient awareness and education is of the utmost importance, as initiation of treatment at the onset of myocardial injury symptoms should be stressed. We believe that any delay in treatment, not just a delay in DTB is likely to increase mortality and comorbidity [13].

\section{References}

[1] Stenestrand, U., Lindback, J. and Wallentin, L. (2006) Outcome of Primary Percutaneous Coronary Intervention VS Prehospital and In-Hospital Thrombolysis for Patients with ST-Elevation Myocardial Infarction. JAMA, 296, 17491756. http://dx.doi.org/10.1001/jama.296.14.1749

[2] Levine, G. (2012) The 2013 STEMI Guideline: Data-Driven Recommendations That Reduce Morbidity and Mortality. Circulation, 1161-1161.

[3] Krumholz, H., Herrin, J. and Miller, L. (2011) Improvements in Door to Balloon Time in the United States 2005 to 2010. Circulation, 124, 1038-1045. http://dx.doi.org/10.1161/CIRCULATIONAHA.111.044107

[4] Menees, D., Peterson, E. and Yongfei, W. (2013) Door-to-Balloon Time and Mortality among Patients Undergoing Primary PCI. The New England Journal of Medicine, 901-909. http://dx.doi.org/10.1056/NEJMoa1208200

[5] Brodie, B., Stuckey, T. and Wall, T. (1998) Importance of Time to Reperfusion for 30-Day and Late Survival and Recovery of Left Ventricular Function after Primary Angioplasty for Acute Myocardial Infarction. The Journal of the American College of Cardiology, 32, 1312-1319. http://dx.doi.org/10.1016/S0735-1097(98)00395-7

[6] Schomig, A., Ndrepepa, G. and Mehilli, J. (2003) Therapy-Dependent Influence of Time-to-Treatment Interval on Myocardial Salvage in Patients with Acute Myocardial Infarction Treated with Coronary Artery Stenting or Thrombolysis. Circulation, 108, 1084-1088. http://dx.doi.org/10.1161/01.CIR.0000086346.32856.9C

[7] Brodie, B., Gersh, B. and Stuckey, T. (2010) When Is Door-to-Balloon Time Critical? Analysis from the HORIZONSAMI (Harmonizing Outcomes with Revascularization and Stents in Acute Myocardial Infarction) and CADILLAC (Controlled Abciximab and Device Investigation to Lower Late Angioplasty Complications) Trials. Journal of American College of Cardiology, 56, 407-413. http://dx.doi.org/10.1016/j.jacc.2010.04.020

[8] Mcnamara, R. and Wang, H. (2006) Effect of Door-to-Balloon Time on Mortality in Patients with ST-Segment Elevation Myocardial Infarction. The Journal of the American College of Cardiology, 47, 2180-2186. http://dx.doi.org/10.1016/j.jacc.2005.12.072

[9] Zijlstra, F., Patel, A. and Jones, M. (2002) Clinical Characteristics and Outcome of Patients with Early (4 h) Presentation Treated by Primary Coronary Angioplasty or Thrombolytic Therapy for Acute Myocardial Infarction. European Heart Journal, 550-557. http://dx.doi.org/10.1053/euhj.2001.2901

[10] Cannon, C.P., Gibson, C.M., et al. (2000) Relationship of Symptom-Onset-to-Balloon Time and Door-to-Balloon Time with Mortality in Patients Undergoing Angioplasty for Acute Myocardial Infarction. The Journal of the American Medical Association, 283, 2941-2947. http://dx.doi.org/10.1001/jama.283.22.2941

[11] Rathore, S., Curtis, J. and Nallamothu, B. (2009) Association of Door-to-Balloon Time and Mortality in Patients $\geq 65$ Years with ST-Elevation Mycoardial Infarction Undergoing Primary Percutaneous Coronary Intervention. The American Journal of Cardiology, 104, 1198-1203. http://dx.doi.org/10.1016/j.amjcard.2009.06.034

[12] Terkelsen, C.J. (2014) Time to Treatment-Door to Balloon Time Is Not Everything. Herz Cardiovascular Disease, 39, 672-676.

[13] Bogaty, P., Lambert, L. and Brophy, J.M. (2014) Door-to-Balloon Time and Mortality. New England Journal of Medicine, 178-182. 